\title{
UTILIZAÇÃO DE ÁGUAS RESIDUÁRIAS PROVENIENTES DO TRATAMENTO BIOLÓGICO DE ESGOTOS DOMÉSTICOS NA PRODUÇÃO DE MUDAS DE Eucalyptus grandis Hill. Ex. Maiden ${ }^{1}$
}

Danielle Camargo Celentano Augusto 2 , Iraê Amaral Guerrini ${ }^{3}$, Vera Lex Engel ${ }^{3}$ e Guillaume Xavier Rousseau ${ }^{4}$

\begin{abstract}
RESUMO - Este trabalho teve por objetivo estudar a viabilidade do uso de águas residuárias provenientes de um sistema biológico de tratamento de esgotos domésticos como alternativa à fertirrigação convencional de viveiros florestais, visando à produção de mudas de Eucalyptus grandis via subirrigação contínua. As variáveis avaliadas foram: altura, diâmetro do coleto, peso da matéria seca da parte aérea e da raiz, área foliar, razão raiz/parte aérea e concentração nutricional foliar. O desenvolvimento das plantas, em geral, foi superior no tratamento convencional com adubos minerais. A razão raiz/parte aérea foi favorecida no tratamento com água residuária. Os resultados indicaram que a água residuária pode ser utilizada na fertirrigação de viveiros para a produção do Eucalyptus grandis, pois todas as plantas cresceram, sem deficiência ou toxidez aparente. Entretanto, constatou-se que essas mudas necessitavam de um maior tempo no viveiro, em comparação com as produzidas com fertilizantes minerais.
\end{abstract}

Palavras-chave: Fertirrigação, água residuária e produção de mudas.

\section{USE OF BIOLOGICALLY TREATED WASTEWATER IN Eucalyptus grandis Hill. Ex. Maiden SEEDLING PRODUCTION BY CONTINUUM SUB IRRIGATION}

\begin{abstract}
This work studied the effect of treated domestic wastewater derived from a biological treatment system as an alternative to nursery conventional fertirrigation for Eucalyptus grandis seedling production. The evaluated variables were: height, basal diameter, shoot and roots dry weight, plant total leaf area, shoot/ root ratio and leaf nutritional status of the seedlings. Greater development of seedlings was found when mineral fertilizers were used, but root/shoot ratio was greater when wastewater was used. The results showed that wastewater can be used as an alternative for conventional mineral fertirrigation, considering that all plants grew, without showing any nutritional deficiency or toxicity symptoms. Seedlings fertirrigated with wastewater, however, spend more time in the nursery, when compared to those produced with mineral fertilizers.
\end{abstract}

Keywords: Fertirrigation, wastewater and seedling production.

\section{INTRODUÇÃO}

A água vem-se tornando uma das maiores preocupações mundiais, dada uma possível crise mundial de abastecimento (POSTEL et al., 1996). Gasi (1988) afirmou que existem diferentes alternativas para a recuperação ou tratamento de águas residuárias de origens doméstica, industrial ou agrícola, muitas delas complexas e onerosas e outras simples e de baixo custo. Como exemplo de uma alternativa simples e de baixo custo, a Fazenda Experimental Lageado da UNESP, em Botucatu, SP, possuía um sistema de coleta e tratamento biológico do esgoto da Comunidade Olaria, utilizando plantas aquáticas. Resultados prévios da avaliação deste sistema indicaram que o emprego de diferentes

\footnotetext{
${ }^{1}$ Recebido em 06.03.2006 e aceito para publicação em 11.03.2007.

${ }_{2}^{2}$ Instituto do Homem e Meio Ambiente da Amazônia. Rua domingos Marreiros, 2020 (Altos), 66060-160 Belém-PA E-mail:danicelentano@yahoo.com.br>.

${ }^{3}$ Faculdade de Ciências Agronômicas/UNESP. Rua José Barbosa de Barros, 1780, 18610-307 Botucatu-SP. Bolsista CNPq.

${ }^{4}$ Embrapa Amazônia Oriental. Travessa Dr. Éneas Pinheiro s/n, 66095-100 Belém-PA. Bolsista CNPq.
} 
plantas aquáticas produziu resultados satisfatórios na remoção de carga poluidora presente no efluente gerado por essa comunidade rural (GUIMARÃES et al., 2000).

Estudos realizados em outros países têm demonstrado a eficiência do uso das águas residuárias na fertirrigação de culturas agrícolas, com a obtenção de excelentes resultados, uma vez que são geralmente ricas em nutrientes (BASTOS, 1999). Pensando numa reutilização futura em larga escala de efluentes, a atividade florestal, por suas peculiaridades, apresenta-se como alternativa promissora, principalmente por não envolver produção de alimentos para consumo humano e nem riscos à saúde (CROMER, 1980). Além disso, por ser realizada em larga escala, tem potencialidade de consumir grande volume de efluentes.

No Brasil, o sistema de irrigação de viveiros mais usual é a microaspersão, sistema que gera grandes desperdícios em razão de fatores como vento, espaços vazios e má distribuição dos aspersores em relação às mudas. A subirrigação contínua se mostra como alternativa para a economia de água, assim como um sistema eficiente para um rápido e controlado desenvolvimento das mudas, e vem sendo utilizada principalmente no manejo de mini e microjardins clonais. Visando ao aproveitamento de águas residuárias, esse sistema se mostra ainda mais propício, pois diminui a possibilidade de contaminação humana e do ambiente.

Augusto et al. (2003) obtiveram resultados satisfatórios estudando a produção de mudas de Croton floribundus Spreng. (Capixingui) e Copaifera langsdorfii. Desf. (copaíba) em um sistema de subirrigação com águas residuárias resultantes do tratamento biológico do esgoto da Comunidade Olaria. Embora as mudas produzidas com essa água residuária tenham apresentado crescimento inferior àquelas produzidas com fertilizantes minerais, não foram constatadas mortalidade, deficiência ou toxidez aparente.

Considerando que o Eucalyptus grandis Hill. Ex. Maiden (eucalipto) é uma das principais espécies florestais exóticas plantadas no Brasil para fins industriais e tem importância estratégica na economia florestal do país, este trabalho teve por objetivo apresentar o resultado da produção de mudas de Eucalyptus grandis utilizando águas residuárias em um sistema de subirrigação contínua.

\section{MATERIAL E MÉTODOS}

Este experimento foi conduzido em 2001 na casa de vegetação do Viveiro Florestal do Departamento de Recursos Naturais da Faculdade de Ciências Agronômicas (FCAUNESP) em Botucatu, SP. As coordenadas geográficas do local são: 22 $2^{\circ} 50^{\prime} \mathrm{S}$ e $48^{\circ} 24^{\prime} \mathrm{W}$. O clima é classificado como $\mathrm{CWb}$ (mesotérmico de inverno seco), com temperaturas médias anuais de $19,5^{\circ} \mathrm{C}$ e pluviosidade anual média de $1.300 \mathrm{~mm}$. A temperatura média na casa de vegetação durante um dia de sol é de $33^{\circ} \mathrm{C}$.

\subsection{Espécie Estudada e Produção das Mudas}

$\mathrm{O}$ experimento foi montado utilizando-se o Eucalyptus grandis Hill. Ex. Maiden. As sementes utilizadas foram procedentes da Cia. Duratex S.A. (Agudos, SP). A semeadura ocorreu em abril de 2001.

Foram utilizados tubetes de polietileno de $120 \mathrm{~mL}$ de capacidade, com substrato constituído de $60 \%$ de casca de Pinus decomposta, $15 \%$ de vermiculita granulometria fina, $25 \%$ de húmus e terra vegetal, cujas análises químicas (Quadro 1) foram realizadas de acordo com a metodologia do Laboratório Nacional de Referência Vegetal (1988) e da Embrapa (1997).

Quadro 1 - Análise química do substrato antes do início e no final do experimento, para o tratamento com água residuária (TR) e para o tratamento convencional (TC)

Table 1 - Substrate chemical analyses before the beginning and at the end of the experiment for wastewater (TR) and conventional fertirrigation (TC) treatments

\begin{tabular}{lrrr}
\hline Variáveis & \multicolumn{2}{c}{ Resultados de base seca $\left(110^{\circ} \mathrm{C}\right)$} \\
\cline { 2 - 4 } & Início & \multicolumn{2}{c}{ Final } \\
\cline { 2 - 4 } & & \multicolumn{2}{c}{$\mathrm{TR} / \mathrm{Kg})$} \\
\hline $\mathrm{N}$ total & 7,10 & 4,00 & 5,00 \\
$\mathrm{P}_{2}$ O5 total & 5,40 & 4,20 & 3,60 \\
$\mathrm{~K}_{2}$ O total & 1,20 & 1,10 & 1,50 \\
Matéria Orgânica & 630,00 & 610,00 & 620,00 \\
Carbono total & 250,00 & 338,90 & 344,50 \\
Ca total & 9,40 & 6,30 & 6,90 \\
Mg total & 6,50 & 5,70 & 8,00 \\
$\mathrm{~S}$ total & 1,10 & 0,40 & 0,80 \\
\hline & & \multicolumn{2}{c}{$\left(\mathrm{mg} \mathrm{L} \mathrm{L}^{-1}\right)$} \\
\hline $\mathrm{Zn}$ total & 114,00 & 68,00 & 96,00 \\
$\mathrm{Mn}$ total & 236,00 & 130,00 & 126,00 \\
$\mathrm{Cu}$ total & 38,00 & 32,00 & 44,00 \\
$\mathrm{Na}$ total & 120,00 & 380,00 & 400,00 \\
Ferro total & 2950,00 & 4300,00 & 5600,00 \\
\hline $\mathrm{pH}$ (CaCl $\left.{ }_{2} 0,01 \mathrm{M}\right)$ & 5,4 & 4,6 & 5,0 \\
\hline Relação C/N & $35 / 1$ & $18 / 1$ & $17 / 1$ \\
\hline
\end{tabular}




\subsection{Delineamento Experimental}

Foi estudado o desenvolvimento do eucalipto sob dois diferentes tratamentos, fertirrigação convencional (TC) e com água residuária (TR), tendo cada tratamento quatro parcelas com 25 plantas cada, sendo consideradas úteis as nove plantas centrais. Os tratamentos foram comparados através do teste t para amostras independentes. Através do teste de Bartlett para homogeneidade de variância, verificou-se que as variâncias dos dados de nitrogênio e potássio foliar não eram homocedásticas (valores discrepantes), havendo, portanto, necessidade de se analisarem esses dados através do teste U de MannWhitney (não paramétrico).

\subsection{Fertirrigação}

A fertirrigação, utilizando tanto água residuária quanto água limpa mais fertilizantes minerais (tratamento convencional), foi realizada via subirrigação, sistema em que os tubetes, dentro de uma bandeja de aço galvanizado, permaneceram continuamente submersos até uma profundidade de $2 \mathrm{~cm}$ da sua base, absorvendo água por capilaridade (Figura 1). O sistema foi controlado por uma bóia, o que proporcionou uma distribuição uniforme da solução para as plantas. Em razão do aparecimento de musgos, em especial no tratamento com água residuária, houve a necessidade de limpeza dos sistemas a cada 14 dias.

A água residuária utilizada foi proveniente da Estação Biológica de Tratamento de Esgotos da Comunidade Rural Olaria, comunidade composta por 15 casas e aproximadamente 65 habitantes. Nessa estação de tratamento biológico, o esgoto doméstico passa por diferentes caixas de fibro-cimento. Nas três primeiras caixas há decantação, separando o material sólido mais grosseiro; na quarta, o material atravessa caixas com brita e terra, onde há melhor filtragem. Em seguida, o resíduo líquido passa por um leito filtrante composto por três partes de casca de arroz e uma parte de solo de textura média, além de diferentes espécies aquáticas (junco, aguapé e taboa), que retiram mais matéria orgânica da água. Guimarães et al. (2000) apresentam esse sistema de tratamento de esgotos de forma detalhada. Segundo eles, a água ao final do tratamento continua não sendo própria ao consumo humano, pois ainda apresenta matéria orgânica e vestígios de coliformes fecais. Aágua utilizada no experimento foi aquela coletada nas saídas das caixas com plantas, última fase do tratamento.
A adubação na fertirrigação convencional foi realizada com nitrato de amônio, nitrato de potássio e monoamônio fosfato (MAP), de acordo com a adubaçãopadrão do viveiro florestal da FCA. Em razão dessa adubação-padrão, foram calculadas as doses de nutrientes adicionadas por tubete, e, a partir desses dados, obtevese o que foi fornecido semanalmente, via fertirrigação, para cada planta: aproximadamente $30 \mathrm{mg}$ de nitrato de potássio, $5 \mathrm{mg}$ de MAP e $25 \mathrm{mg}$ de nitrato de amônio.

Análises químicas e de condutividade elétrica da água de ambos os tratamentos (Quadro 2) foram feitas a cada 15 dias, de acordo com o Laboratório Nacional de Referência Vegetal (1988), e a uma adaptação da Embrapa (1997) realizada pelo Laboratório de Análises de Plantas e Solos do Departamento de Recursos Naturais - FCA/UNESP.

\subsection{Coleta e Análise dos Dados}

Os parâmetros altura e diâmetro de coleto das mudas foram avaliados quinzenalmente, durante três meses, com o uso de régua graduada e paquímetro digital, respectivamente. A área foliar foi medida ao final do experimento ( 3 meses), utilizando-se o aparelho AAM8 Hayoshi Denkoh, bem como a produção de massa seca da parte aérea e das raízes das plantas úteis de cada parcela.
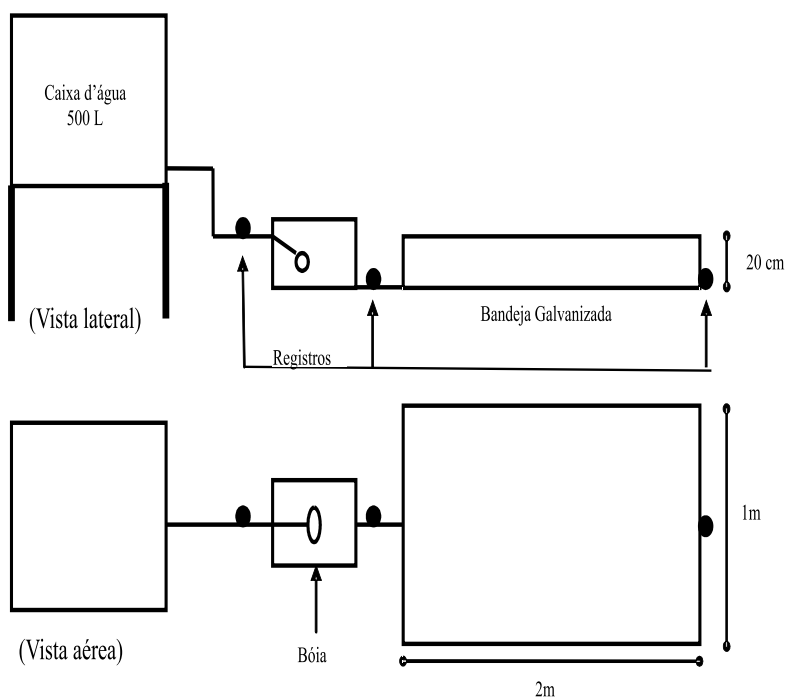

Figura 1 - Sistema de subirrigação contínua construído para este experimento.

Figure 1 - Continuum subirrigation system built up for this experiment.

R. Árvore, Viçosa-MG, v.31, n.4, p.745-751, 2007 
Quadro 2 - Análises química e de condutividade elétrica (C.E.) da água do tratamento com água residuária (TR) e do tratamento convencional (TC)

Table 2 - Chemical analyses and electrical conductivity (C.E.) for wastewater (TR) and conventional (TC) treatments

\begin{tabular}{|c|c|c|c|c|c|c|c|c|c|c|c|c|c|}
\hline & $\mathrm{N}$ & $\mathrm{P}$ & $\mathrm{K}$ & $\mathrm{Ca}$ & $\mathrm{Mg}$ & $\mathrm{S}$ & $\mathrm{Na}$ & $\mathrm{Cu}$ & $\mathrm{Fe}$ & $\mathrm{Mn}$ & $\mathrm{Zn}$ & \multirow{2}{*}{$\begin{array}{c}\mathrm{pH} \\
\mathrm{CaCl}_{2}\end{array}$} & \multirow{2}{*}{$\begin{array}{l}\text { C.E. } \\
\text { dS } / \mathrm{m}\end{array}$} \\
\hline & & & & & & & $(\mathrm{mg} / \mathrm{l})$ & & & & & & \\
\hline $\mathrm{TR}^{1}$ & 161,70 & 6,20 & 23,20 & 29,10 & 4,80 & 6,30 & 48,10 & 0,01 & 2,60 & 0,10 & 0,10 & 7,10 & 0,70 \\
\hline $\mathrm{TC}^{2}$ & 229,00 & 15,10 & 119,30 & - & - & - & - & - & - & - & - & 7,00 & 1,90 \\
\hline
\end{tabular}

${ }^{1}$ Média de nove amostragens ao longo do experimento e ${ }^{2}$ Média de sete amostragens ao longo do experimento.

Foram feitas análises químicas de macro e micronutrientes nos tecidos foliares das mudas, a fim de avaliar o estado nutricional das plantas ao final do experimento, de acordo com a metodologia de Malavolta et al. (1997). A partir dos resultados desta análise, foi calculado o acúmulo de nutrientes nas folhas.

\section{RESULTADOS E DISCUSSÃO}

\subsection{Medição da altura total e do diâmetro de coleto}

O desenvolvimento em altura e diâmetro das mudas de eucalipto foi significativamente superior no tratamento convencional (Figura 2) ao final do experimento (95 dias), fato ocorrido provavelmente pela maior concentração de macronutrientes na solução nutritiva em relação à água residuária. Ao final do experimento, pode-se constatar, também, que as mudas apresentaram alturas superiores àquelas sugeridas para plantio (> $30 \mathrm{~cm}$ ), devido ao crescimento surpreendente nos últimos 30 dias de viveiro no tratamento convencional.

\subsection{Peso da Matéria Seca e Área Foliar}

O sistema de subirrigação utilizado neste experimento favoreceu o desenvolvimento de sistemas radiculares vigorosos e sem o enovelamento em ambos os tratamentos. Em função dessa volumosa produção de raízes, principalmente no tratamento com água residuária, foi necessário fazer podas periódicas e simultâneas daquelas que saíam do tubete em direção à água. De acordo com Mafia et al. (2005), a qualidade do sistema radicular tem implicação direta com a mortalidade das mudas no campo e conseqüente onerosidade do empreendimento.

A área foliar e o peso seco da parte aérea e da raiz (dentro do tubete) evidenciaram significativa superioridade no tratamento convencional (Quadro 3), sendo $107,8 \%, 120,1 \%$ e $38,6 \%$ superiores, respectivamente, em relação ao tratamento com água residuária. O peso seco das raízes totais (raiz no tubete mais raiz podada ao longo do experimento) não apresentou diferença estatística entre os tratamentos. As raízes das mudas do tratamento com água residuária saíam do tubete em busca de maior disponibilidade de nutrientes.

A razão raiz/parte aérea foi favorecida no tratamento com água residuária (TR), o que pode representar uma característica desejável para maior equilíbrio mecânico das mudas em condições de campo.

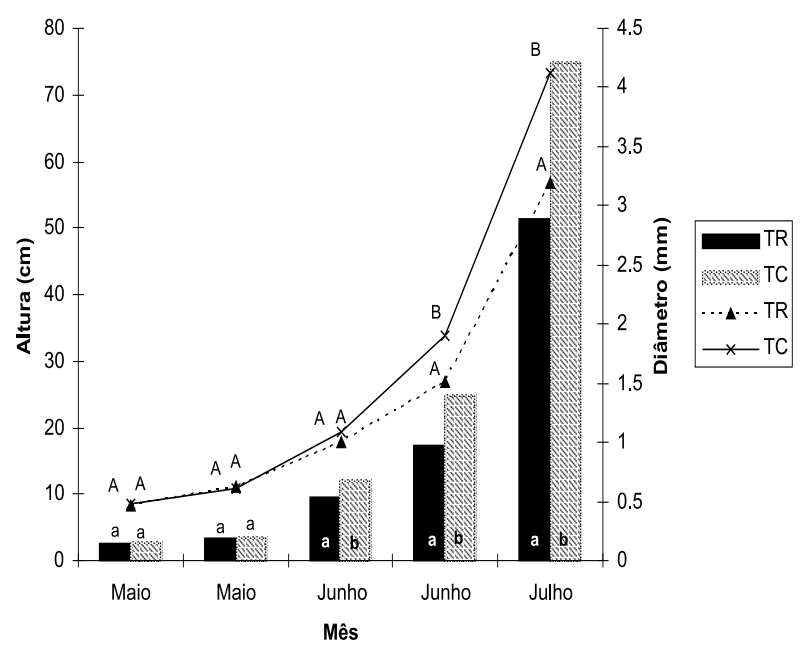

Figura 2 - Médias de altura (colunas) e diâmetro do coleto (linhas) para o tratamento com água residuária (TR) e para o tratamento convencional (TC). Médias seguidas de mesma letra não apresentam diferenças estatísticas, segundo o teste t, para amostras independentes a $5 \%$ de probabilidade $(\mathrm{p}<0,05)$.

Figure 2 - Height (column) and basal diameter (lines) means for wastewater (TR) and conventional (TC) treatments. Means followed by the same letter are not significantly different at $5 \%$ probability level $(p<0.05)$. 
Quadro 3 - Médias de área foliar, peso da matéria seca da parte aérea e da raiz e razão raiz/ parte aérea das mudas de eucalipto para o tratamento com água residuária (TR) e para o tratamento convencional (TC). Letras iguais significam que não há diferenças estatísticas segundo o teste t para amostras independentes a $5 \%$ de probabilidade(p $<0,05)$

Table 3 - Leaf area, shoot and root dry weight and root/shoot ratio means for wastewater (TR) and conventional (TC) treatments. Values followed by the same letters are not statistically different at $5 \%$ probability level $(p<0.05)$ by the t- test

\begin{tabular}{|c|c|c|c|c|c|c|}
\hline & \multirow[t]{2}{*}{ Área Foliar } & \multicolumn{3}{|c|}{ Matéria Seca (g/muda) } & \multicolumn{2}{|c|}{ Razão Raiz/Parte Aérea } \\
\hline & & Parte Aérea & Raiz no Tubete & Raiz Total ${ }^{\mathbf{1}}$ & (Raiz no Tubete) & $(\text { Raiz Total })^{1}$ \\
\hline TR & 326,41 a & 2,79 a & $0,57 \mathrm{a}$ & $0,76 \mathrm{a}$ & $0,21 \mathrm{~b}$ & $0,28 \mathrm{~b}$ \\
\hline $\mathrm{TC}$ & $678,46 \mathrm{~b}$ & $6,14 b$ & $0,79 \mathrm{~b}$ & $0,80 \mathrm{a}$ & $0,14 \mathrm{a}$ & $0,14 \mathrm{a}$ \\
\hline
\end{tabular}

${ }^{1}$ Raiz total é a soma do peso de matéria seca das raízes no tubete e daquelas podadas durante o experimento.

\subsection{Avaliação do Estado Nutricional das Folhas}

Os resultados apontaram valores significativamente maiores nas folhas do tratamento convencional nos teores de N, P, K e Mn (Quadro 4). O maior teor de $\mathrm{N}, \mathrm{P}$ e K nas folhas do tratamento convencional já era esperado em função da adubação mineral semanal, cujos teores são altos quando comparados com o da água residuária. Esses macronutrientes são os principais elementos exigidos pelas plantas, o que certamente causou o melhor desempenho destas no tratamento convencional para os parâmetros avaliados. Já o alto valor de Mn surpreendeu, pois não houve micronutrientes nessa adubação convencional, a não ser os existentes originalmente na água e no substrato.

Quanto ao estado nutricional das plantas, segundo Silveira e Higashi (1998), os teores de N e K no tratamento convencional podem ser considerados adequados, enquanto os do tratamento com água residuária são baixos. Entretanto, ainda segundo esses autores, o teor de $\mathrm{P}$ no tratamento convencional pode ser classificado como alto (excessivo), enquanto no tratamento com água residuária se mostrou adequado. De acordo com resultados de referência sobre o estado nutricional do Eucalyptus (SILVEIRA et al., 2000), os teores de $\mathrm{N}$, P e $\mathrm{K}$ nos tecidos foliares das plantas do tratamento com água residuária, embora sejam baixos, não se encontram na faixa de deficiência.

Os tecidos foliares das mudas do tratamento com água residuária apresentaram significativamente maiores teores de $\mathrm{Fe}$ e $\mathrm{Zn}$, provavelmente em razão do alto teor desses elementos nessa água. Durante o desenvolvimento do ensaio, não houve mortalidade e, tampouco, o aparecimento de sintomas de deficiência e de toxidez nesse tratamento.
A adição de fertilizantes à água residuária para a obtenção de maior produtividade deve ser estudada no futuro. A adubação da água residuária poderá resultar em plantas ainda mais vigorosas e saudáveis que essas produzidas neste experimento. Além disso, efluentes com maiores concentrações de nutrientes, como esgotos (que não passam por filtros com plantas aquáticas) e rios poluídos, podem representar uma alternativa para a produção florestal, seja em viveiros ou na irrigação de campo. Augusto et al. (2004) estudaram o crescimento de mudas de eucalipto subirrigadas com águas poluídas do rio Lavapés (Botucatu, SP) e encontraram resultados muito favoráveis no uso dessa água para a produção dessa espécie.

\subsection{Considerações sobre o Sistema de Subirrigação Contínua}

Neste sistema, pelas suas dimensões $(100 \mathrm{~cm}$ de comprimento, $200 \mathrm{~cm}$ de largura e $2 \mathrm{~cm}$ de lâmina de água) e tempo necessário para as plantas utilizarem toda a água nele contida (aproximadamente quatro dias, considerando o consumo pelas plantas mais a água evaporada), tem-se que o gasto de água diário por planta é de 33,3 mL, em média. Comparando com os dados obtidos em viveiros comerciais (dados obtidos em 2001), que utilizam o sistema de microaspersão, no qual cada planta gasta em média $83 \mathrm{~mL}$, tem-se que esse sistema de subirrigação proporcionou uma economia de água de aproximadamente $60 \%$. Deve-se considerar também a economia de energia elétrica, a qual representa $4 \%$ do preço final da muda em viveiros comerciais (dados fornecidos em 2001 por viveiros comerciais), e a economia de fertilizantes utilizados na fertirrigação.

R. Árvore, Viçosa-MG, v.31, n.4, p.745-751, 2007 
Quadro 4 - Análise química foliar (médias) e acúmulo de nutrientes nas folhas para o tratamento com água residuária (TR) e para o tratamento convencional (TC). Médias seguidas de letras iguais não apresentam diferenças estatísticas, segundo o teste $t$, para amostras independentes a $5 \%$ de probabilidade $(\mathrm{p}<0,05)$. Os resultados referentes ao acúmulo de nutrientes não foram comparados estatisticamente

Table 4- Nutrient leaf status (means) and leaf accumulated nutrients for wastewater (TR) and conventional (TC) treatments. Values with same letters are not significantly different at $5 \%$ level $(p<0.05)$ by the $t$-test. Data on leaf-accumulated nutrients were not compared

\begin{tabular}{|c|c|c|c|c|}
\hline \multirow{2}{*}{$\begin{array}{l}\text { Macronutrientes } \\
\left(\mathrm{g} \mathrm{kg}^{-1}\right)\end{array}$} & \multicolumn{2}{|c|}{ Análise Química Foliar } & \multicolumn{2}{|c|}{ Acúmulo de Nutrientes nas Folhas ( $\left.\mathrm{mg} \mathrm{muda}^{-1}\right)$} \\
\hline & TC & $\mathrm{TR}$ & $\mathrm{TC}$ & TR \\
\hline N & $19,25 \mathrm{a}$ & $26,75 \mathrm{~b}$ & 26,37 & 69,02 \\
\hline $\mathrm{P}$ & $2,35 \mathrm{a}$ & $2,78 \mathrm{~b}$ & 3,22 & 7,17 \\
\hline $\mathrm{K}$ & $11,25 \mathrm{a}$ & $17,25 \mathrm{~b}$ & 15,41 & 44,51 \\
\hline $\mathrm{Ca}$ & $7,75 \mathrm{a}$ & $7,50 \mathrm{a}$ & 10,62 & 19,35 \\
\hline $\mathrm{Mg}$ & $2,97 \mathrm{a}$ & $2,77 \mathrm{a}$ & 4,08 & 7,16 \\
\hline $\mathrm{S}$ & $1,80 \mathrm{a}$ & $2,10 \mathrm{a}$ & 2,47 & 5,42 \\
\hline \multicolumn{5}{|l|}{$\begin{array}{l}\text { Micronutrientes } \\
\left.(\mathrm{mg}) \mathrm{kg}^{-1}\right)\end{array}$} \\
\hline $\mathrm{B}$ & 59,25 a & $53,00 \mathrm{a}$ & 0,07 & 0,15 \\
\hline $\mathrm{Cu}$ & 7,00 a & $8,50 \mathrm{a}$ & 0,01 & 0,02 \\
\hline $\mathrm{Fe}$ & $229,25 \mathrm{~b}$ & $167,00 \mathrm{a}$ & 0,31 & 0,43 \\
\hline Mn & $126,50 \mathrm{a}$ & $162,00 \mathrm{~b}$ & 0,17 & 0,41 \\
\hline $\mathrm{Zn}$ & $131,75 \mathrm{~b}$ & $55,00 \mathrm{a}$ & 0,18 & 0,14 \\
\hline
\end{tabular}

Além disso, outros fatores devem ser relevados: a água utilizada em viveiros comerciais geralmente é limpa e, muitas vezes, potável; o desperdício causado pelo sistema de aspersão se tornará inaceitável em função da escassez deste recurso prevista para o futuro. Estudos visando desenvolver e viabilizar o sistema de subirrigação para a produção florestal em larga escala devem ser apoiados e difundidos, sobretudo se possibilitarem o uso de águas residuárias para seu funcionamento.

\section{CONCLUSÕES}

Os resultados indicaram que águas residuárias provenientes de sistemas biológicos de tratamento de esgotos podem ser utilizadas na fertirrigação de viveiros para a produção de mudas de Eucalyptus grandis, pois todas as plantas sobreviveram, sem deficiência ou toxidez de nutrientes aparente. Entretanto, constatou-se um menor desenvolvimento geral das mudas produzidas com água residuária quando comparadas com aquelas produzidas com fertilizantes químicos, em razão da menor disponibilidade de macronutrientes, evidenciando que essas mudas necessitarão de maior tempo no viveiro, quando comparadas com as produzidas com fertilizantes minerais.

R. Árvore, Viçosa-MG, v.31, n.4, p.745-751, 2007
O sistema de subirrigação contínua desenvolvido para este experimento mostrou-se promissor para a produção de eucalipto, pois houve excelente adaptação e desenvolvimento desta espécie, além de proporcionar economia de água, fertilizante e energia elétrica. O crescimento das mudas, principalmente no tratamento com fertilizantes químicos, foi excelente e o desenvolvimento do sistema radicular das plantas, favorecido.

\section{AGRADECIMENTOS}

À FAPESP, pelo apoio financeiro (Bolsa de Iniciação Científica, Processo 00/14827-4); aos funcionários da FCA Cláudio R. da Silva, Aparecido A. de Arruda, Manuel Lyra, João M. Rodrigues, Ieda F. Rodrigues, José Carlos De Pieri e José Carlos Coelho e aos profissionais Magali Ribeiro, Rodrigo Trigueiro, Carlos Breda e Jeane Almeida, por toda colaboração no decorrer do experimento.

\section{REFERÊNCIAS}

AUGUSTO, D. C. C.; ENGEL, V. L.; VELINI, E. D. Preliminary results of a floating subirrigation system ("Aquaforest system") as an alternative to conventional tree nurseries. Ecological Engineering, v.22, p.61-66, 2004. 
AUGUSTO, D. C. C. et al. Utilização de esgotos domésticos tratados através de um sistema biológico na produção de mudas de Croton floribundus Spreng. (Capixingui) e Copaifera langsdorfii. Desf. (Copaíba). Revista Árvore, v.27, n.3, p.335-342, 2003.

BASTOS, R. K. X. Fertirrigação com águas residuárias. In: FOLEGATTI, M. V. (Coord.) Fertirrigação: citrus, flores e hortaliças. Guaiba: Agropecuária, 1999. 279p.

CROMER, R. N. Irrigation of radiata pine with wastewater: A review of the potential for tree growth and water renovation. Australian Forest, v.43, p.87-100, 1980.

EMPRESA BRASILEIRA DE PESQUISA AGROPECUÁRIA - EMBRAPA. Centro Nacional de Pesquisa de Solos. Manual de método de análises de solo. 2.ed. Rio de Janeiro, 1997. 212p. (Embrapa-CNPS. Documentos, 1).

GASI, T. M. T. Opções para tratamento de esgotos de pequenas comunidades. São Paulo: CETESB, 1988. 36p. (Série Manuais, 3)

GUIMARÃES, A. B. et al. Treatment of domestic wastewaters in rural zone by using aquatic plant system. In: INTERNATIONAL SYMPOSIUM ON SANITARY AND ENVIRONMENTAL ENGINEERING, 2000, Trento. Proceedings... Trento: Hyper, 2000. p.237-243.
LABORATÓRIO NACIONALDEREFERENCIA VEGETAL - LNRV. Análise de corretivos, fertilizantes e inoculantes. Métodos oficiais. Brasília: Ministério da Agricultura, Secretaria Nacional de Defesa Agropecuária., 1988.

MAFIA, R. G. et al. Critério técnico para determinação da idade ótima de mudas de eucalipto para plantio. Revista Árvore, v.29, n.6, p.947-953, 2005.

MALAVOLTA, E.; VITTI, G. C.; OLIVEIRA, S. A. Avaliação de estado nutricional das plantas : princípios e aplicações. 2.ed. Piracicaba: Associação Brasileira para a Pesquisa da Potassa e do Fosfato, 1997. 319p.

POSTEL, S. L.; DAILY, G. C.; EHRLICH, P. R. Human appropriation of renewable fresh water. Science, v.271, p.785-788, 1996.

SILVEIRA, R. L. V. A.; HIGASHI, E. N. Produção de mudas de Eucalyptus spp. na Lwarcel. Piracicaba: IPEF. 1998. 75p. (Relatório Final - Convenio Lwarcel IPEF RR Agroflorestal SC Ltda.).

SILVEIRA, L. V. A. S. et al. Avaliação do estado nutricional do Eucalyptus: diagnose visual, foliar e suas interpretações. In: GONÇALVES, J. L. M.; BENEDETTI, V. (Eds.) Nutrição e fertilização florestal. Piracicaba: IPEF, 2000. p.80-102. 
